\title{
Behavior of class-level landscape metrics across gradients of class aggregation and area
}

\author{
Maile C. Neel ${ }^{1,2, *}$, Kevin McGarigal ${ }^{1}$ and Samuel A. Cushman ${ }^{1,3}$ \\ ${ }^{1}$ Department of Natural Resources Conservation, Holdsworth Natural Resources Center, University of \\ Massachusetts, Amherst, Massachusetts 01003, USA; ${ }^{2}$ Current address: Department of Natural Resource \\ Sciences and Landscape Architecture 2116 Plant Sciences Building, University of Maryland, College Park, MD \\ 20742, USA; ${ }^{3}$ Current address: USDA Forest Service, Rocky Mountain Research Station, Missoula MT 59807, \\ USA; *Author for correspondence (e-mail: mneel@umd.edu)
}

Received 15 October 2002; accepted in revised form 18 November 2003

Key words: Connectivity, Fragmentation, Landscape pattern analysis, Neutral landscape models, FRAGSTATS

\begin{abstract}
Habitat loss and fragmentation processes strongly affect biodiversity conservation in landscapes undergoing anthropogenic land use changes. Many attempts have been made to use landscape structure metrics to quantify the independent and joint effects of these processes. Unfortunately, ecological interpretation of those metrics has been plagued by lack of thorough understanding of their theoretical behavior. We explored behavior of 50 metrics in neutral landscapes across a 21-step gradient in aggregation and a 19-step gradient in area using a full factorial design with 100 replicates of each of the 399 combinations of the two factors to assess how well metrics reflected changes in landscape structure. Metric values from real landscapes were used to determine the extent of neutral landscape space that is represented in real landscapes. We grouped metrics into three major behavioral classes: strongly related to focal class area $(n=15)$, strongly related to aggregation $(n=7)$, and jointly responding to area and aggregation $(n=28)$. Metrics strongly related to class area exhibited a variety of distinct behaviors, and many of these metrics have unique interpretations that make each of them particularly useful in certain applications. Metrics strongly related to aggregation, independent of class area, are particularly useful in assessing effects of fragmentation. Moreover, metrics in this group exhibited a range of specific behaviors, highlighting subtle but different aspects of landscape aggregation even though we controlled only one aspect of aggregation. The non-linear behavior exhibited by many metrics renders interpretation difficult and use of linear analytical techniques inappropriate under many circumstances. Ultimately, comprehensive characterization of landscapes undergoing habitat loss and fragmentation will require using several metrics distributed across behavioral groups.
\end{abstract}

\section{Introduction}

Because of the fundamental reciprocal relationships between landscape structure and ecological processes, objectively quantifying spatial landscape structure remains an important aspect of landscape ecology (Turner 1989). A large number of metrics and indices have been developed to characterize landscape composition and configuration based on categorical map patterns (e.g., McGarigal and Marks 1995; McGari- gal et al. 2002). These metrics are used to analyze landscape structure for a wide variety of applications, including quantifying landscape change over time (O'Neill et al. 1997) and relating structure to ecosystem (Wickham et al. 2000), population and metapopulation processes (Kareiva and Wennergren 1995; Fahrig 2002). Arguably the major application of landscape structure metrics has been assessing effects of habitat loss and fragmentation on landscape connectivity (e.g., Fahrig and Merriam 1985; With et al. 
1997; Fahrig 1998; Fahrig and Jonsen 1998; Wickham et al. 1999; Riitters et al. 2000).

Fragmentation is a complex phenomenon that can be seen both as a consequence of habitat loss and as a process in and of itself (McGarigal and McComb 1995). The process of fragmentation of a continuous matrix habitat begins with reduction in habitat area and an increase in proportion of edge-influenced habitat. Initially, the majority of the remaining habitat may continue to be connected but will become increasingly perforated and incised (Jaeger 2000). When sufficient habitat is lost, or when the original habitat occurs as a patch mosaic rather than a matrix ( 50-60\% of the landscape), remaining habitat often becomes separated into isolated patches (Jaeger 2000). In random landscapes, patch isolation typically increases most dramatically in the vicinity of the percolation threshold (Gardner et al. 1987). As habitat loss continues, some of the isolated patches will themselves be broken up into varying sized fragments with a peak in the number of patches occurring when the focal class comprises $15-30 \%$ of the landscape. Eventually, some fragmented patches will be lost and others will shrink, further isolating remnant patches. The point along this gradient at which the landscape actually becomes fragmented depends on the organism or process of interest. Throughout the fragmentation process, many aspects of landscape composition and configuration are affected including patch area, number of patches, amount of patch edge, patch shape complexity, interpatch distances, and adjacency of like cells; all of these characteristics affect landscape connectivity. Further, they are affected in different ways in different parts of the fragmentation gradient. Given this complexity, it is not surprising that a large number of metrics have been used to describe landscape responses to fragmentation and a number of metrics have been developed specifically for the purpose of assessing changes in landscape connectivity resulting from fragmentation [e.g., aggregation index (He et al. 2000); landscape division, effective mesh size, and splitting index (Jaeger 2000); clumpiness index (McGarigal et al. 2002), cohesion (Schumaker 1996), and contagion ( $\mathrm{Li}$ and Reynolds 1993)].

The diversity of metrics available and the complexity of habitat loss and fragmentation effects make it difficult to choose an appropriate metric or suite of metrics for a particular situation. Ultimately, metric selection should be based on the hypotheses being tested, on aspects of landscape structure that are relevant to the organism or process of interest, and on characteristics of the landscape itself (e.g., whether the focal class occurs as disjunct patches or as the matrix) (McGarigal and Marks 1995). Informed metric selection requires thorough understanding of the conceptual and computational basis of each metric. Meaningful interpretation of the metrics also requires a relevant landscape classification at an appropriate scale for a particular organism or process (Gustafson 1998; McGarigal 2002). Jaeger (2000) has proposed eight criteria to assess metric suitability for assessing fragmentation: intuitive interpretation, low sensitivity to small patches, monotonic reaction to different fragmentation phases, detection of structural differences, mathematical simplicity, modest data requirements, mathematical homogeneity, and additivity. An additional desirable metric attribute is ability to assess changes in landscape configuration that are independent of area. This ability is particularly important for studies of habitat fragmentation in which disentangling effects of loss of habitat area from effects of changes in configuration (e.g., aggregation, subdivision, and isolation) of the remaining habitat has been a major goal (e.g., Fahrig and Merriam 1985; Bender et al. 1998; Fahrig and Jonsen 1998; Trzcinski et al. 1999; Belisle and Clair 2002).

We argue that ecological interpretation of landscape structure also requires understanding of expected behavior of landscape structure metrics under controlled conditions. Expected behavior of a number of metrics has been examined across gradients in focal class area (Gustafson and Parker 1992; Riitters et al. 1995; Hargis et al. 1997; Gustafson 1998; Hargis et al. 1998). A subset of these studies have also examined metric behavior under a limited array of different types of spatial dispersion (e.g., Hargis et al. 1997; Gustafson 1998; Hargis et al. 1998). Additionally, Saura and Martínez-Millán (2000) illustrated the behavior of four metrics (Cohesion, Number of Patches, Total Edge, and Area-Weighted Mean Shape Index) across gradients of area and levels of fragmentation. Despite these efforts, metric behavior across gradients in both aggregation and area has not been explored simultaneously for most metrics. The purpose of the present work is to explore behavior of 55 class-level metrics (Table 1) for binary neutral landscapes across a 21-step gradient from low to maximum class aggregation and a 19-step gradient in class area. It is essential to note that the only aspect of configuration gradient we explicitly controlled was the degree of class aggregation. Thus, evaluation of the behavior of metrics designed to measure other as- 


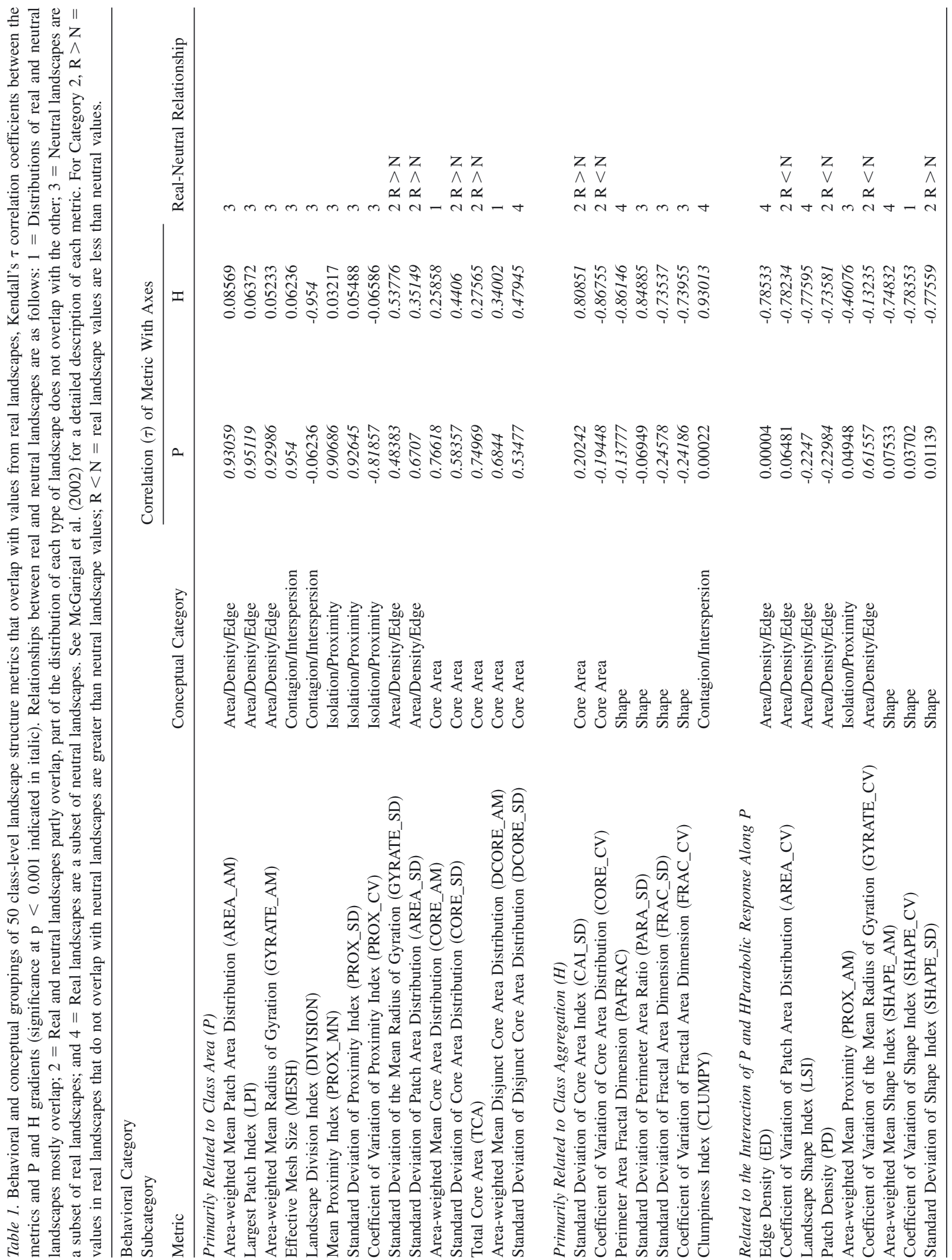




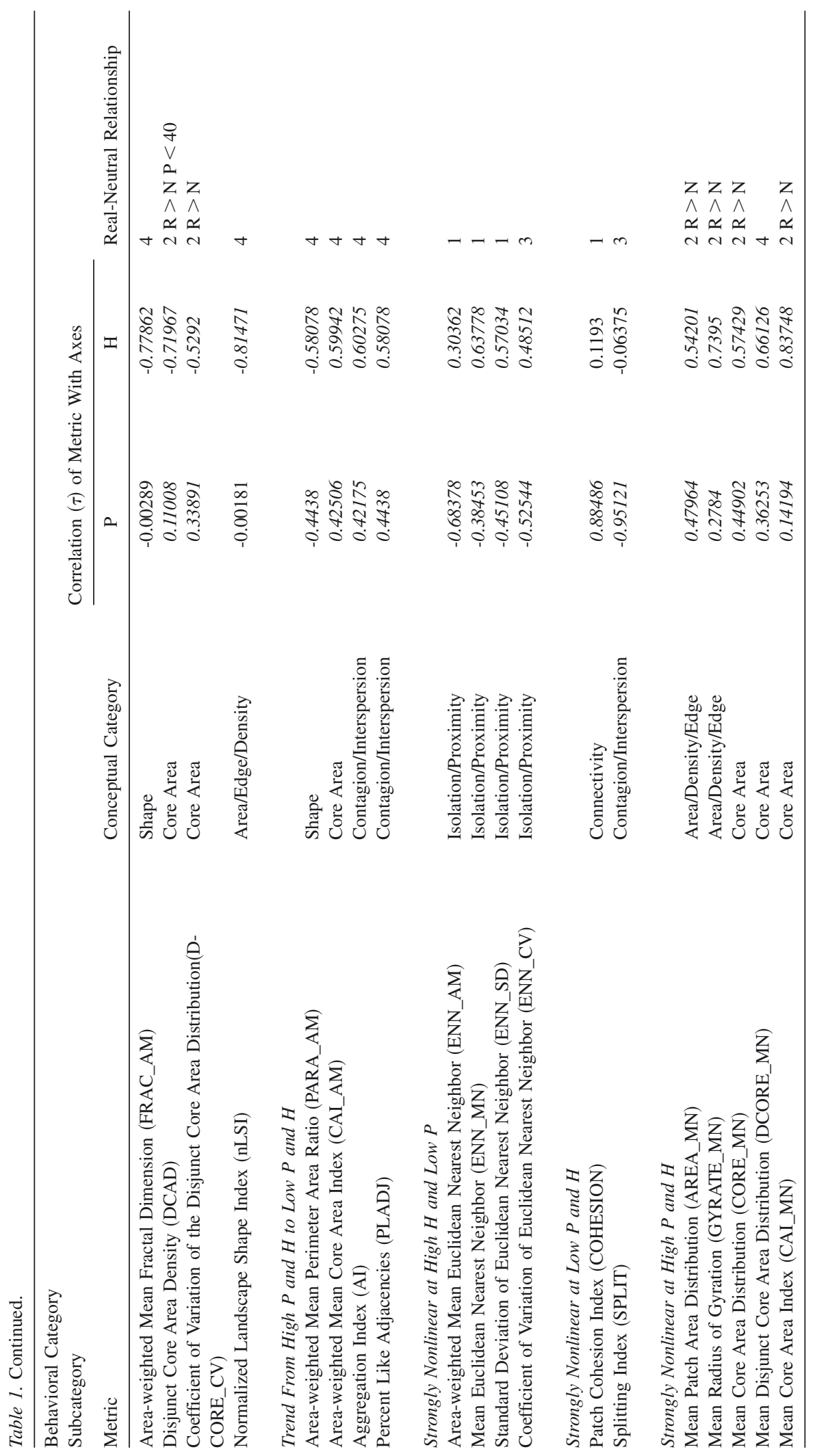



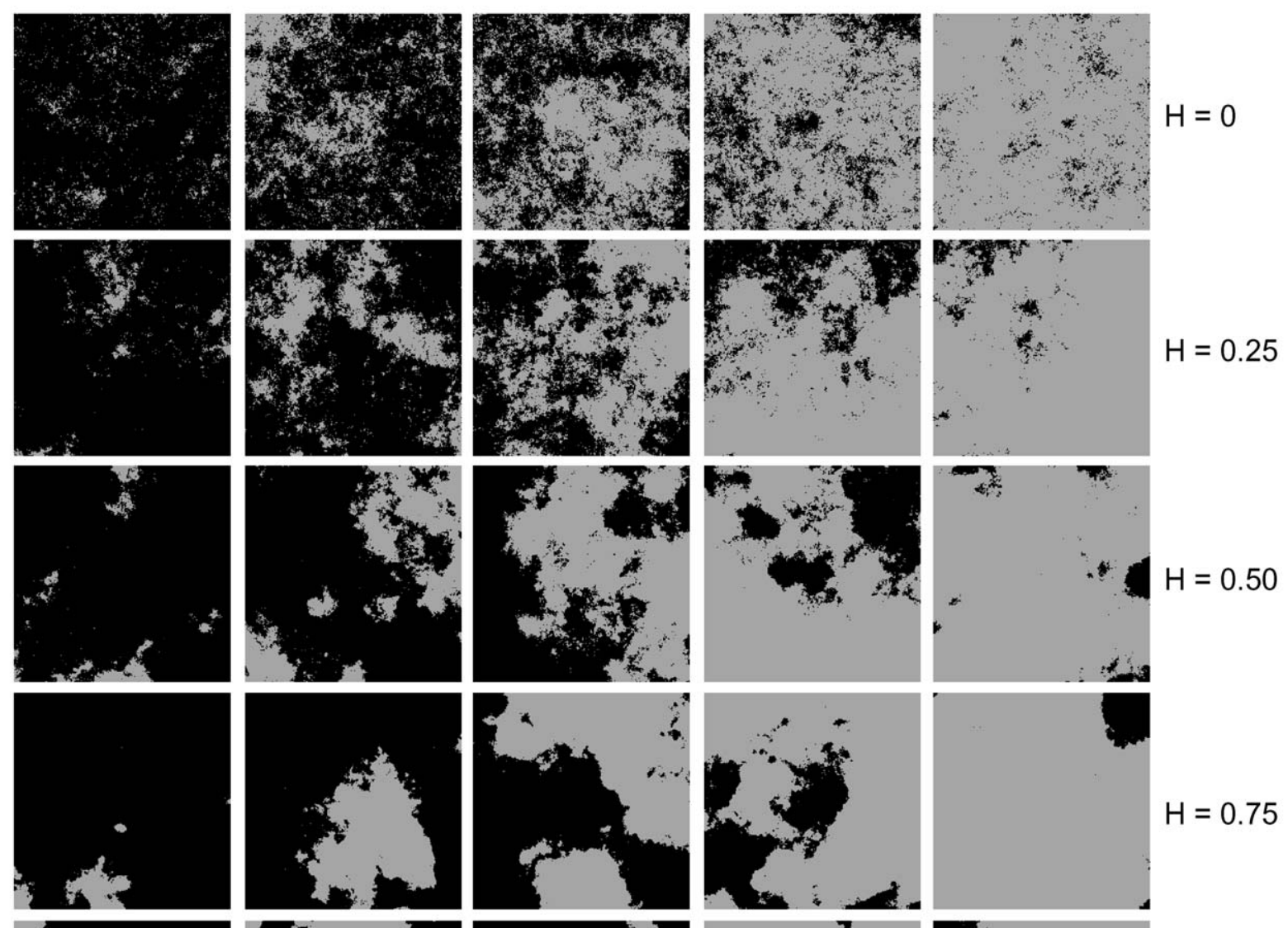

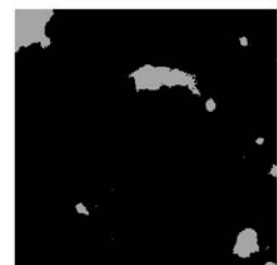

$\mathrm{P}=95$

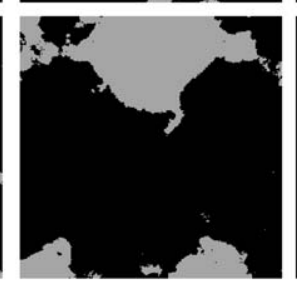

$\mathrm{P}=75$

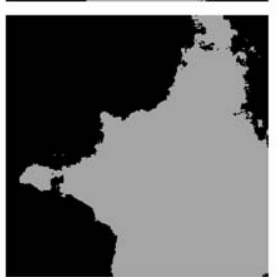

$\mathrm{P}=50$

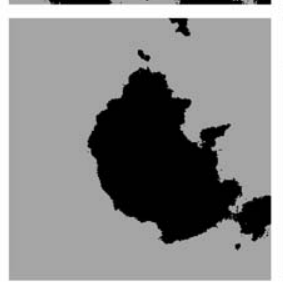

$\mathrm{P}=25$

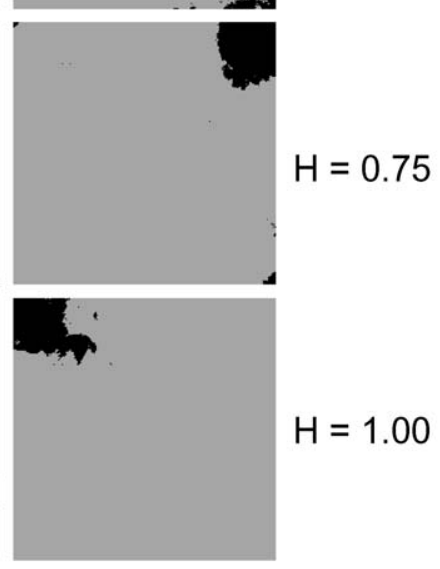

$\mathrm{P}=5$

Figure 1. Example neutral landscapes representing the range of aggregation and area gradients represented in the study. Gradient orientation is the same as the orientation in graphs representing metric behavior (Figure 3-6).

pects of configuration was constrained by the degree to which they covaried with aggregation in the neutral models. The focus on class aggregation was due in part to practical limitations (i.e., inability to create neutral landscape gradients reflecting other aspects of configuration with available software), but also to the fact that changes in class aggregation are a major component, if not the dominant component, of the fragmentation process.

\section{Methods}

We used the computer program RULE (Gardner $1999)$ to generate $256 \times 256$ cell, binary multi fractal neutral landscapes (Figure 1). RULE uses the midpoint displacement algorithm (Saupé 1988) to generate multi fractal maps where $\mathrm{H}$, the degree of spatial autocorrelation among adjacent cells, controls the degree of aggregation in the landscape. We generated landscapes in a full factorial design across a 21-step gradient in aggregation $(\mathrm{H}=0-1$ in 0.05 increments) and a 19-step gradient in focal class area 
$(\mathrm{P}=5-95 \%$ in 5\% increments) with 100 replicate landscapes for each of the 399 factor combinations (resulting in a total of 39,900 landscapes).

Neutral landscape models generate grids of cells that are comparable to categorical, raster-based representations of real landscapes commonly used in landscape structure analysis. These maps have proven useful as null models for a wide array of landscape ecological investigations (Gardner et al. 1987), including developing spatially explicit meta- population and source-sink models (With et al. 1997; Haydon and Pianka 1999; With and King 2001) and identifying fragmentation thresholds (With et al. 1997). A major benefit of neutral landscapes is the ability to strictly and independently control both area included in a user-specified number of habitat classes and the degree of spatial aggregation of those classes (Gardner et al. 1987). Neutral landscapes, however, have their limitations. In particular, they can represent only a limited range of the complex spatial patterns that are typically found in real landscapes (e.g., they do not adequately represent linear features such as rivers and the associated riparian habitats) and they do not provide a way to vary the degree of aggregation of habitat patches, only the degree of aggregation of individual cells. Schumaker (1996) has criticized neutral landscapes as being too simplistic to be relevant to structure measurement in real landscapes. However, his conclusion on this matter was based on inappropriate comparisons of spatially random neutral landscapes with real landscapes. For our purposes, the level of control over area and aggregation afforded by neutral landscapes provides insight into sensitivity of metrics to variation in landscape composition and configuration that would otherwise remain obscured (Gustafson 1998).

We calculated 55 class-level landscape structure metrics (Table 1) for each landscape using the computer program FRAGSTATS 3.2 (McGarigal et al. 2002). Some of these metrics are calculated based on aggregate properties of the class using all cells of the focal class without reference to individual patches. Others are calculated for each patch and then values for all patches of the focal class are summarized. We included four formulations for the patch-based metrics: mean value $(\mathrm{MN})$, area-weighted mean value (AM), standard deviation (SD) and coefficient of variation $(\mathrm{CV})$. For organizational purposes, metrics have previously been grouped into classes based on conceptual similarity corresponding largely to the aspect of landscape structure emphasized, but also to some degree to similarity in computational approach. These conceptual groups correspond to those described in FRAGSTATS and include area/density/ edge, shape, core area, isolation/proximity, contrast, contagion/interspersion, and connectivity (McGarigal et al. 2002).

While neutral landscapes have no associated dimensions, we imposed dimensions that allowed us to directly compare results from a set of real landscapes described below. Metric calculations were based on a $30 \mathrm{~m}$ cell size, a $90 \mathrm{~m}$ (i.e., 3 cell) edge depth, a $500 \mathrm{~m}$ search radius, and an eight-neighbor rule (for patch delineation). Landscape boundary was not included as edge in calculations where its inclusion was optional and no border was specified. Our choice of cell size was arbitrary and primarily affects the magnitude of the class metrics, not the shape of their distributions. The exact value of edge depth was also arbitrary but was chosen to fall within the range of edge depth effects documented for different organisms in forested landscapes (e.g., Chen et al. 1995; Demaynadier and Hunter 1998; Gehlhausen et al. 2000). Edge depth magnitude affects all metrics related to core area. A smaller edge depth value would result in more patches with core areas and more core area for a given patch size with core, while a larger edge depth value would have the opposite effect. The search radius affects only metrics that are based on the distribution of patches within a specified distance of a focal patch; for our purposes, only the proximity index is affected.

We used the programming language Perl to create input scripts for RULE and batch files for FRAGSTATS, to manage the RULE and FRAGSTATS output files, and to calculate summary statistics (minimum, maximum, mean, sample standard deviation, and sample coefficient of variation) for FRAGSTATS results from replicate samples within each of the 399 combinations of aggregation and area. Results were plotted on three dimensional surface graphs using MATLAB 6.1(The MathWorks 2001) such that mean values for each metric at each $\mathrm{H} \times \mathrm{P}$ combination are indicated by the height of the surface on the Z-axis.

We also investigated relationships between metric values realized in neutral landscapes with those from all classes in 428 real landscapes (2727 landscape $\mathrm{x}$ class combinations) from three disjunct regions of North America. The first region included an approximately $15,000 \mathrm{~km}^{2}$ portion of western Massachusetts that is dominated by northern hardwood forests and characterized by rolling hills, agricultural valleys and 
scattered urban and residential development. The landcover map used in this analysis was created from 1999 aerial photography with a minimum mapping unit of 0.1 acre for MassGIS, an office of the Massachusetts Executive Office of Environmental Affairs. We converted the original vector coverage into a grid with a $30 \mathrm{~m}$ cell size and reclassified it into seven cover classes to ensure adequate distribution of classes across sub-landscapes (see below). The seven classes included forest, water, grassland, cropland, urban, high-density residential, and low-density residential.

The second region was the $8,480 \mathrm{~km}^{2}$ San Juan National Forest in southwestern Colorado. This mountainous landscape has rugged topography and extreme elevational relief. The landcover is zonal, with Ponderosa pine (Pinus ponderosa) forest in the lower elevations, mixed-coniferous and aspen (Рориlus tremuloides) forest in the middle elevations, and spruce-fir forest (primarily Picea engelmannii and Abies lasiocarpa) and treeless alpine communities at the highest elevations. The landcover map used in this analysis was derived from the USDA Forest Service Integrated Resources Inventory (IRI) and Resources Information System (RIS) database. The landcover map was developed by the Forest Service from a combination of information sources and processes, including the existing RIS polygon boundaries, aerial photo interpretation (1993, 1:24000 natural color), digital image analysis of Landsat Thematic Mapper imagery, and logic written into a $\mathrm{C}$ program to delineate and attribute polygons. We converted the vector coverage into a grid with a $25 \mathrm{~m}$ cell size and reclassified it into four cover classes to ensure adequate distribution of all classes across sub-landscapes (see below). The four classes included forest, water, riparian, and non-forested.

The third region was an approximately $20,000 \mathrm{~km}^{2}$ area of central Idaho. This region is also mountainous with zonal landcover. The landcover map used in this analysis was developed by the Idaho Gap Analysis project with 30 meter pixels. We reclassified the map into five cover classes, including forest, rock, riparian, grass, and shrub with the same goal of ensuring adequate distribution of all classes across sub-landscapes (see below).

We clipped non-overlapping sub-landscapes each of the three regional landcover maps using a square grid 256 cells per side. This process resulted in 155 sample landscapes for western Massachusetts, 152 sample landscapes for the San Juan National Forest, and 221 for central Idaho.

Results from real and neutral landscapes could not be compared directly because, while there is a value of $\mathrm{P}$ for both types of landscapes, real landscapes have no value of $\mathrm{H}$. To assess the range of $\mathrm{H}$ occupied by real landscapes, we superimposed metric values from both landscapes types (Figure 2). Within the neutral landscape space, we recorded the range of $\mathrm{H}$ values associated with metric values from real landscapes at each value of P. In several cases the majority of values from real landscapes occupied a restricted part of the $\mathrm{H}$ gradient while a relatively small proportion of landscapes occupied a broader range. We noted the portion of the $\mathrm{H}$ gradient with high densities of real landscape values separately from areas with only a few landscapes to be conservative in assessing application of our results in real landscapes. The levels of aggregation represented by both high and low densities of real landscapes were then plotted on the surface of the three-dimensional graphs of metric behavior. This plotting format allows simultaneous illustration of the general magnitude of all metrics across $\mathrm{H} \times \mathrm{P}$ space and assessment of the portion of that space found in real landscapes. We also examined the nature of overlap between real and neutral landscapes. Our comparisons of real and neutral landscapes yielded five types of relationships: 1) real and neutral landscapes mostly overlapping; 2) real and neutral landscapes partially overlapping; 3) neutral landscapes being a subset of real landscapes; 4) real landscapes being a subset of neutral landscapes; and 5) real and neutral landscapes not overlapping. Five metrics (FRAC_MN, PARA_MN, PARA_CV, SHAPE_MN, and CAI_CV) fell into this latter category and were eliminated from further analysis yielding a total of 50 metrics examined in detail.

We calculated pair-wise Pearson product moment correlation coefficients among all pairs of metrics using PROC CORR in the computer program SAS (SAS Institute 1999) to compare the degree of similarity among metric surfaces across the aggregation and area gradients (Robinson and Bryson 1957). By correlating metric values at each of the sampled points on each surface, we were able to estimate of the degree of correspondence or overall similarity between the surfaces. Some authors (Robinson 1962; Merriam and Sneath 1966) have suggested a slight modification of the Pearson's correlation coefficient to account for dependencies among points on a grid. 


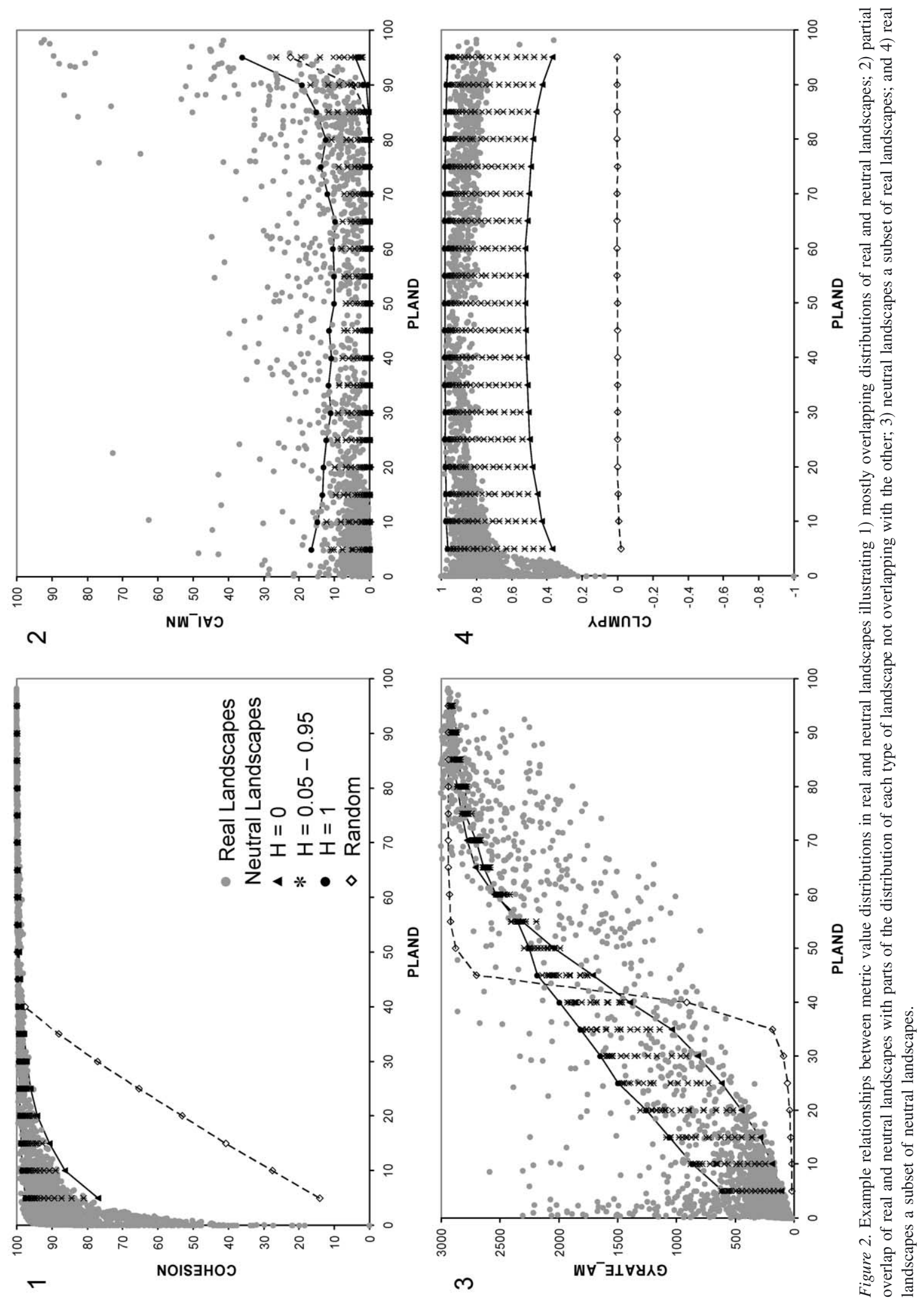


We used both approaches to calculate correlations between a subset of metrics and found the results to be identical so we used Pearson product-moment correlation for computational simplicity. Correlation coefficients were squared and subtracted from 1 to convert them to pairwise distances. The matrix of pairwise distances among all metrics was used in a hierarchical agglomerative cluster analysis using Ward's linkage method in PROC CLUSTER (SAS Institute 1999). We then used the results from the cluster analysis and visual inspection of the surfaces to assign metrics to behavioral groups. We used PROC CORR to calculate Kendall's $\tau$ correlation coefficients between each metric and the $\mathrm{H}$ and $\mathrm{P}$ gradients.

\section{Results and discussion}

We recognized three broad behavioral groups of metrics: (1) metrics that were primarily related to $\mathrm{P}$ and relatively independent of $\mathrm{H}(\mathrm{n}=15)$, (2) metrics that were primarily related to $\mathrm{H}$ and relatively independent of $P(n=7)$, and (3) metrics that were related to the interaction between $\mathrm{H}$ and $\mathrm{P}$, many with dramatically nonlinear behavior at extreme values of $\mathrm{P}$ or $\mathrm{H}$ $(\mathrm{n}=28)$ (Table 1). This third major group contains five loosely defined subgroups of metrics based on the nature the interaction between $\mathrm{P}$ and $\mathrm{H}$. We used these groups to identify general similarities among metrics that affect their interpretation. There of course is substantial variation within groups so it is still important to thoroughly understand each metric.

Metric values in real and neutral landscapes were mostly overlapping in 7 metrics and were partially overlapping in 16 metrics (Table 1). Values in neutral landscapes were a subset of those in real landscapes for 14 metrics and real landscape values were a subset of neutral landscape values for 13 metrics. (Table 1). As mentioned previously, values in real and neutral landscapes did not overlap at all for five metrics. In addition to identifying five types of relationships between real and neutral landscapes (Figure 2, Table 1), we recognized three categories of overlap within neutral landscape space itself: 1) neutral metric values not represented in real landscapes; 2) neutral metric values well represented in real landscapes; and 3) neutral metric values represented in relatively few real landscapes (i.e., outliers). While this approach provides some insight into metric behavior in real landscapes it is limited in two ways. First, more of the $\mathrm{H} \times \mathrm{P}$ space would likely be represented if we looked at more real landscapes. Second, we can only look in detail at behavior within neutral landscape space, we have no way to address metric behavior in real landscapes that falls outside this space.

\section{Metrics primarily related to class area $(P)$}

Fifteen metrics in four conceptual groups were predominantly related to $\mathrm{P}$ (Table 1), although there was considerable variability in behavior among metrics (Figure 3). Because metrics in this group exhibited a variety of distinct behavioral patterns, it was difficult to clearly distinguish subgroups. Of the metrics in this group, LPI, AREA_AM, GYRATE_AM, DIVISION, and MESH were most strongly and clearly related to P, with Kendall's $\tau$ correlations all exceeding 0.93 ( $p$ $\leq 0.001$ for all) (Table 1); these metrics were also highly correlated with one another $(r \geq 0.95, \mathrm{p}<$ 0.001 in all cases). As such, in many cases these metrics will not provide substantial information that is not already provided by PLAND-which is easy to interpret. None of the metrics in this group, however, were perfectly correlated with PLAND, and the subtle differences may be important or enlightening under certain circumstances. Additionally, many of these metrics have unique interpretations that make each of them particularly useful.

For example, DIVISION and MESH have intuitive appeal in that they represent the probability that two individuals that are limited to the focal habitat and placed in different areas of a landscape will find each other (Jaeger 2000). Specifically, DIVISION is the probability that two randomly chosen places in a region will not be found in the same undissected area and MESH represents the size $\left(\mathrm{m}^{2}\right)$ of patches that would result if a landscape was divided into equal size patches with the same degree of landscape division as the measured landscape (Jaeger 2000). $\mathrm{MESH}$, it turns out, is functionally equivalent to AREA_AM at the class level; these two metrics differ in magnitude as a function of $P$ (McGarigal et al. 2002) which results in only slightly different behavior across the $\mathrm{H} \times \mathrm{P}$ space. Because DIVISION, MESH and AREA_AM are largely redundant, they would not be used together; however, each can have utility due to their different interpretations and units. Similarly, GYRATE_AM (also known as correlation length) is a useful metric for assessing connectivity in that it can be interpreted directly as the distance that an organism that is placed and moves randomly can traverse 

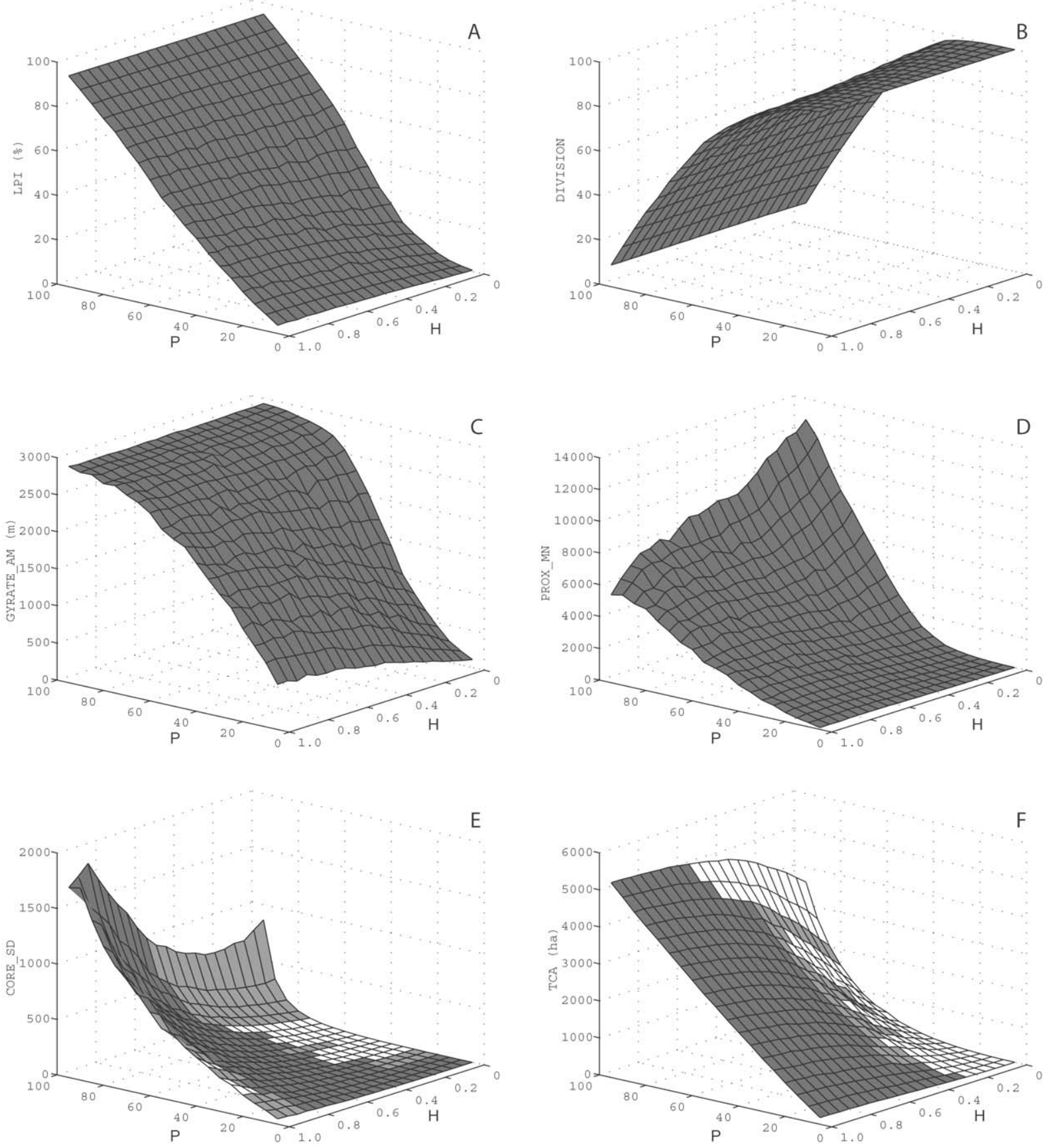

High Density of Real Landscapes

Low Density of Real Landscapes

No Real Landscapes

Figure 3. Three-dimensional plots of 6 of the 15 metrics that are primarily related to class area. Surface height indicates the mean metric value for 100 replicate runs for each $\mathrm{H} \times \mathrm{P}$ combination. Shading indicates the portion of the neutral gradient represented by values in real landscapes. The metric surface grids represent each step in the area and aggregation gradients. Full metric names are provided in Table 1.

and remain in the focal habitat (Keitt et al. 1997). It is important to note that just because these metrics were largely redundant with $\mathrm{P}$ over the $\mathrm{H} \times \mathrm{P}$ gradients we investigated, it does not mean that these met- 
rics will always be so redundant when other or more complex configuration gradients are sampled in either neutral or real landscapes. In addition, even though these metrics were strongly related to $\mathrm{P}$, they exhibited distinct but subtle gradient responses to $\mathrm{H}$. Because the magnitude of the $\mathrm{H}$ response was very small compared to the $\mathrm{P}$ response, it was largely unnoticeable in the surface plots (Figure 3).

Several metrics in this group (AREA_AM, LPI, GYRATE_AM, PROX_MN, and PROX_SD) exhibited distinct asymmetry in behavior at low versus high values of $\mathrm{H}$ across the $\mathrm{P}$ gradient, although the details varied. Specifically, they increased more or less linearly with increasing focal class area at high $\mathrm{H}$, but exhibited nonlinear behavior associated with the percolation threshold (0.41) (Stauffer 1985) at low $\mathrm{H}$ (Figure 3A, 3C, and 3D). The percolation threshold effect, even at the lowest $\mathrm{H}$ level, was dampened overall compared with the effect in random landscapes (Gustafson and Parker 1992; Hargis et al. 1998), and the threshold effect diminished rapidly as $\mathrm{H}$ increased. Additionally, we detected a second unexpected transition at approximately the percolation threshold for these metrics. Below this point, metric values for any $\mathrm{P}$ were larger at high $\mathrm{H}$ than at low $\mathrm{H}$; the trend was reversed above $\mathrm{P}=\sim 45 \%$. This gradient reversal is important to recognize because the interpretation of these metrics with regards to class aggregation is different when $\mathrm{P}$ is above versus below the percolation threshold. Three core area metrics in this group (TCA, CORE_AM, and DCORE_AM) also exhibited asymmetry in behavior at low versus high values of $\mathrm{H}$ across the $\mathrm{P}$ gradient similar to the metrics described above, but the asymmetry was much more pronounced and the nonlinear behavior at low $\mathrm{H}$ occurred at a much higher $\mathrm{P}$ than the percolation threshold (e.g., Figure 3F).

Finally, several metrics in this group (AREA_SD, CORE_SD, GYRATE_SD, PROX_SD, and DCAD_SD) were predominantly related to the P gradient over much of their ranges, but exhibited nonlinear or, in some cases, erratic behavior at very high values of $P$ (e.g., Figure $3 E$ ). The first three of these metrics had a distinctly parabolic relationship along the $\mathrm{H}$ gradient at high $\mathrm{P}$, while the latter three metrics had less straightforward but nevertheless dramatic changes in behavior at high $\mathrm{P}$ (primarily when values of $\mathrm{H}$ were either $>0.8$ or $<0.3$ ). Most of these metrics measure variability in the spatial character of patches and reflect the increasing variability among patches as $\mathrm{H}$ and $\mathrm{P}$ increase. Specifically, as $\mathrm{H}$ increases and the class becomes more aggregated, some patches become larger and more extensive. However, even at the highest $\mathrm{H}$, some cell clusters remain small and disjunct. Thus, the absolute variability among patches increases with $\mathrm{H}$. Similarly, as $\mathrm{P}$ increases and the class becomes more common, some patches naturally coalesce into larger and more extensive patches; yet some patches remain small and disjunct, which increases the absolute variability among patches.

Neutral landscape space was well represented by real landscapes for ten of these metrics, however neutral landscapes represented the range of real landscape behavior for only two of these metrics (CORE_AM and DCORE_AM) (Table 1, Figure 3AD). In all cases where values from real landscapes lie outside the neutral landscape space the real landscape values are larger. It is not possible to assess whether the metric behavior outside the neutral landscape space is the same as that within the space. Values of the remaining five metrics from real landscapes occupy only a portion of the neutral landscape space, typically the higher levels of aggregation (Figure 3EF).

\section{Metrics primarily related to class aggregation $(H)$}

Seven metrics in three conceptual groups were predominantly related to $\mathrm{H}$ and were mostly independent of class area (Table 1, Figure 4), although there was considerable variability in behavior among metrics in terms of the degree of interaction. These metrics are particularly useful in assessing the aggregation component of fragmentation without the need for post-hoc analyses to separate these oft confounded effects. All of these metrics were highly correlated with the $\mathrm{H}$ gradient with correlation coefficients ranging in magnitude from $\tau=0.73(\mathrm{p}<0.001)$ for FRAC_SD to $\tau=0.93$ ( $\mathrm{p}<0.0001)$ for CLUMPY. It is noteworthy that several metrics purported to be measures of class aggregation [e.g., aggregation index (He et al. 2000), cohesion (Schumaker 1996), and landscape shape index (McGarigal et al. 2002)] did not fall into this behavioral subgroup.

Except for the clumpiness index (CLUMPY), which is based on like cell adjacencies, all of the metrics in this subgroup are related to some aspect of patch shape complexity (Table 1). FRAC_SD, FRAC_CV, PAFRAC, and PARA_SD are based on perimeter area relationships. Although often not realized by practitioners, the relationship between shape 

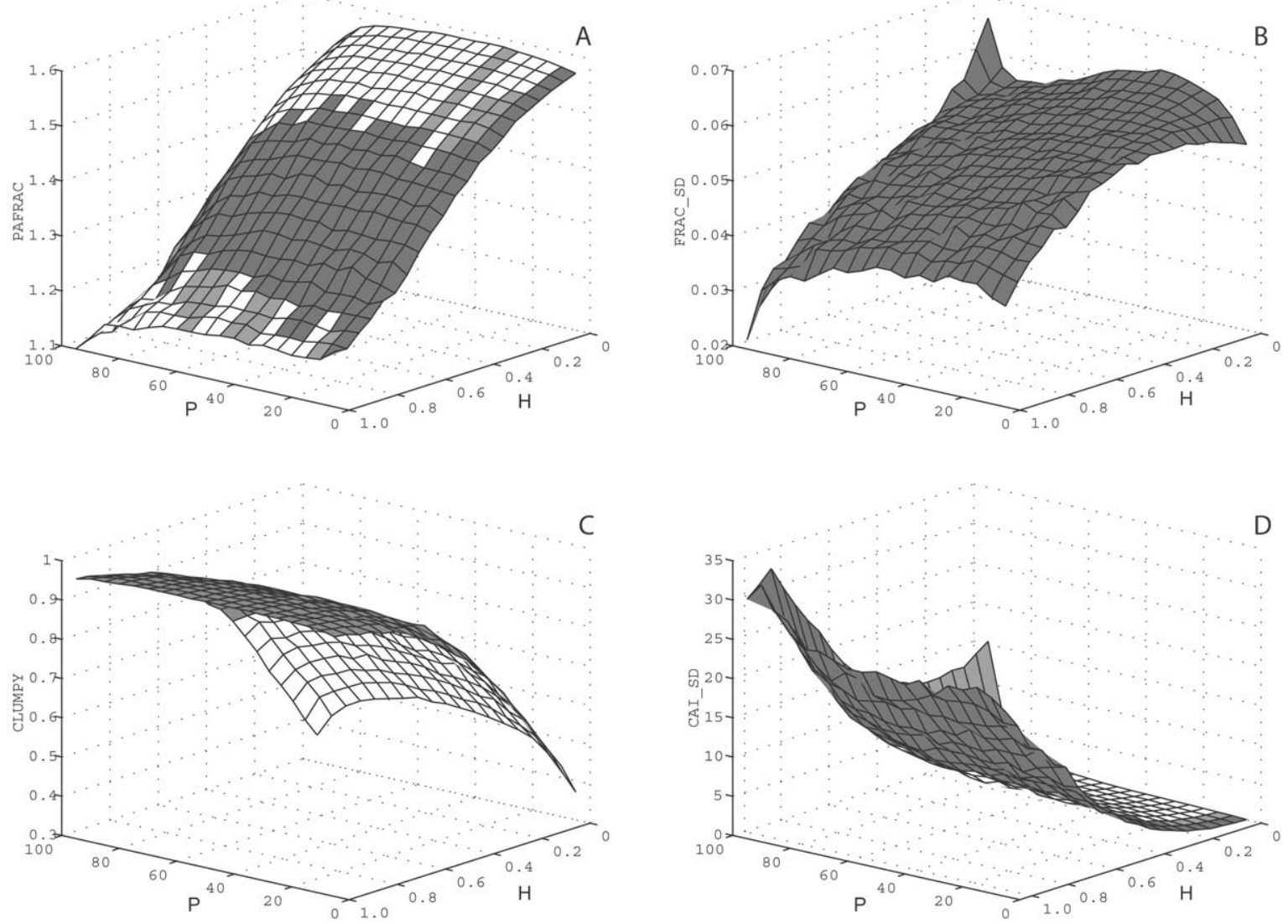

High Density of Real Landscapes

Low Density of Real Landscapes

No Real Landscapes

Figure 4. Four of the seven metrics that are primarily related to class aggregation and are relatively independent of class area. Surface height indicates the mean metric value for 100 replicate runs for each $\mathrm{H} \times \mathrm{P}$ combination. Shading indicates the portion of the neutral gradient represented by values in real landscapes. The metric surface grids represent each step in the area and aggregation gradients. Full metric names are provided in Table 1.

complexity based on perimeter-area relationships and class aggregation is well established (e.g., Bogaert 2002) and thus this behavioral similarity is to be expected. However, many other metrics based on class aggregation do not behave similarly. While the core metrics in this subgroup (CORE_CV, CAI_SD) are not calculated using like adjacencies or perimeter area ratios, the amount of core area in a patch is strongly affected by patch shape, with more convoluted patches having less core area when size is equal.

The behavior of this group of metrics is well illustrated by PAFRAC, which is a measure of the relative rate of increase of patch area and perimeter. Shape complexity increases (i.e., patches become increasingly plane filling) with decreasing levels of aggregation, and this relationship is largely consistent across all levels of P (Figure 4A). In fact, PAFRAC is relatively unaffected by changes in class area when $5 \%<\mathrm{P}<80 \%$. The abrupt decline in PAFRAC across all levels of aggregation when $\mathrm{P}>80 \%$ is likely a statistical artifact. Because PAFRAC is calculated by regressing the log of patch perimeter onto the log of patch area, it is sensitive to sample size. As $\mathrm{P}$ increases above $80 \%$, the number of patches becomes very small and estimates of the metric become unreliable. In landscape ecological studies, PAFRAC has been used primarily as a method for characterizing patch shape complexity (Krummel et al. 1987; Milne 1988; Turner and Ruscher 1988; Iverson 1989; Ripple et al. 1991), not habitat aggregation. Specifically, it describes the power relationship between patch area and perimeter, and thus 
describes how patch perimeter increases per unit increase in patch area. If, for example, small and large patches alike have simple geometric shapes, then PAFRAC will be relatively low, indicating that patch perimeter increases relatively slowly as patch area increases. Conversely, if small and large patches have complex shapes, then PAFRAC will be much higher, indicating that patch perimeter increases more rapidly as patch area increases-reflecting a consistency of complex patch shapes across spatial scales. If, on the other hand, the relative complexity of patch shapes differs substantially between smaller patches and larger patches, then PAFRAC should be estimated separately for each range of patch sizes. The fractal dimension of patch shapes, therefore, is suggestive of a common ecological process or anthropogenic influence affecting patches across a wide range of scales, and differences between landscapes or scale ranges can suggest differences in the underlying pattern-generating process (Krummel et al. 1987). Our results suggest that PAFRAC also provides a means of effectively discriminating among landscapes on the basis of class aggregation and may therefore have even greater utility than previously realized. We should note, however, that the program we used to create the neutral landscapes used in this study was designed to generate fractal landscapes. Thus, perhaps it should not be surprising that PAFRAC behaved so nicely in discriminating among landscapes with varying fractal dimensions. It should be noted, however, that other fractal metrics (FRAC_AM, FRAC_SD, and FRAC_CV) did not behave similarly.

CLUMPY (Figure 4C) was designed explicitly as a measure of class aggregation. Specifically, it measures how aggregated the focal class is by comparing the observed proportion of like cell adjacencies with the proportion expected under a spatially random distribution. CLUMPY ranges from 1 (maximally aggregated) to -1 (maximally dispersed), with 0 indicating a completely spatially random distribution of the focal class. Another metric that is in the parabolic group, nLSI (Figure 5F), also was designed to measure class aggregation and is based on a standardized perimeter-area relationship and is normalized to range from 0 (maximally aggregated) to 1 (maximally dispersed). Both metrics are highly correlated with $\mathrm{H}(\tau$ $=0.93, \mathrm{p}<0.0001$ for CLUMPY and $\tau=-0.87$, $\mathrm{p}<0.0001$ for nLSI) and thus hold promise as measures of class aggregation largely independent of class area. However, CLUMPY is substantially less parabolic than nLSI and as a result may be more eas- ily interpreted (Figure 4). A detailed description of the theoretical behavior and application of these metrics is the subject of an ongoing study and will be reported in a subsequent paper.

Two metrics in this group were coefficients of variation and two were standard deviations of metric values across all patches (Table 1) and thus their interpretation is entirely intuitive but can be useful in providing information regarding among-patch variability in landscapes. Their interpretation is further complicated by the fact that they behave somewhat inconsistently. For example, variability among patches in perimeter-area ratio (PARA_SD) and core area (CAI_SD) increase with increasing aggregation, whereas variability in patch fractal dimension (FRAC_SD,FRAC_CV) decreases with increasing aggregation (Figure 4). Because these metrics are all based directly or indirectly on perimeter-area relationships, it is not clear why they should behave differently.

Metrics in this group had a variety of real landscape-neutral landscape relationships (Table 1). CLUMPY and PAFRAC were the most interpretable in that values from real landscapes fell completely within the range of neutral landscapes values. In the case of CLUMPY, all real landscapes but one had values comparable to $\mathrm{H} \geq 0.30$ (Figure $4 \mathrm{C}$ ). In contrast, PAFRAC values in real landscapes tended to be less extreme than in neutral landscapes (Figure 4A). The entire neutral landscape space was represented in real landscapes for PARA_SD, FRAC_SD, and FRAC_CV, but patches in real landscapes exhibited higher variation then neutral landscapes. This difference is likely a function both of differences in patch shape complexity and in the number of patches in the two types of landscapes. Values of the two remaining metrics (CAI_SD and CORE_CV) partially overlapped in the two landscape types, but differed in the direction of the deviation (Table 1).

\section{Metrics related to the interaction of class area $(P)$ and class aggregation $(H)$}

Twenty-eight metrics in six conceptual groups were related to the interaction between $\mathrm{H}$ and $\mathrm{P}$. We recognized three loosely defined subgroups based on the nature the interaction. One subgroup was distinguished by being related to $\mathrm{H}$ and having parabolic distributions along the $\mathrm{P}$ gradient (Figure 5); one subgroup had a trend from high $\mathrm{H}$ and $\mathrm{P}$ to low $\mathrm{H}$ and $\mathrm{P}$ (Figure 6A-B); and the final subgroup exhibited dra- 

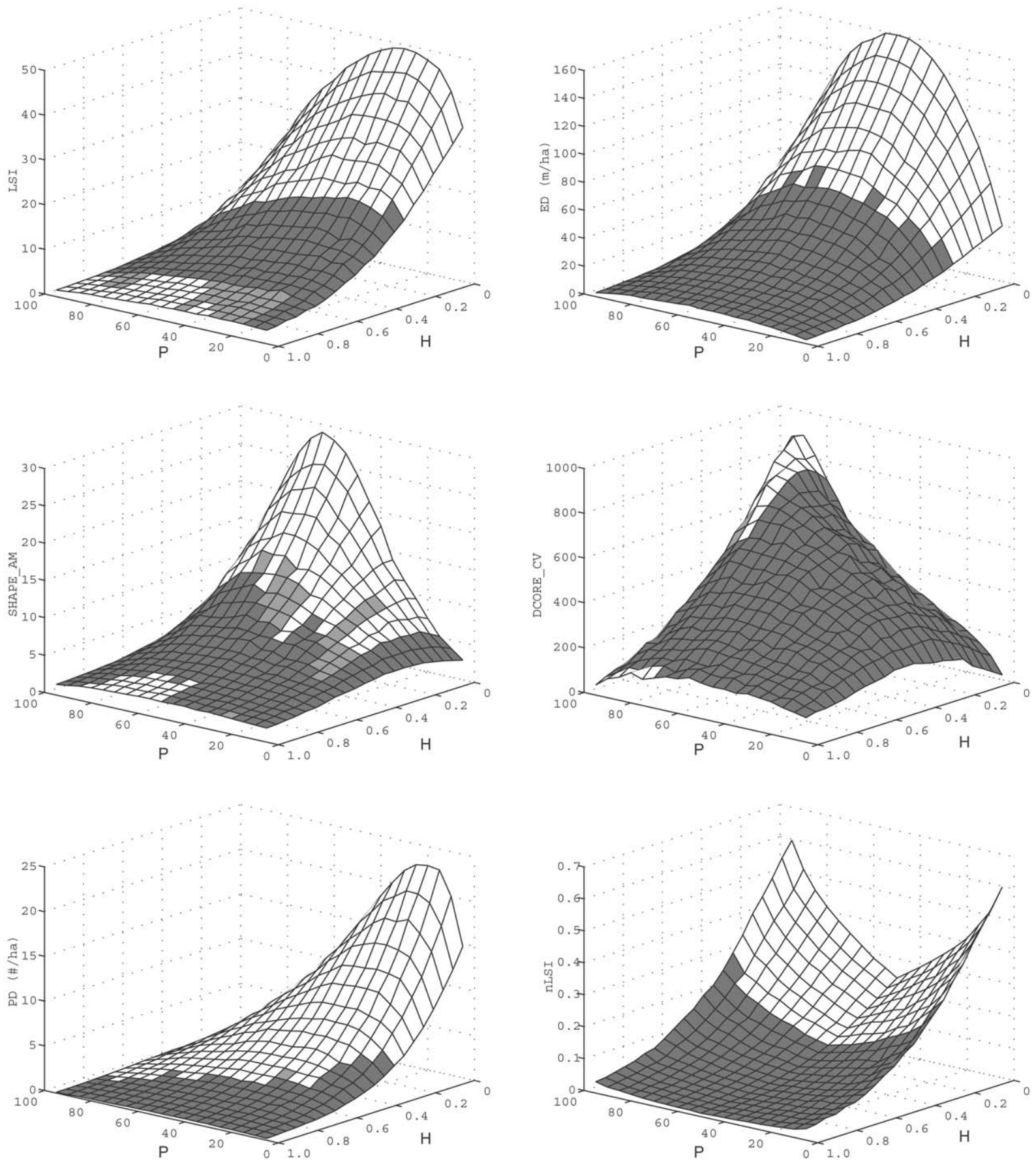

High Density of Real Landscapes

Low Density of Real Landscapes

No Real Landscapes

Figure 5. Three-dimensional plots of 6 of the 13 metrics with parabolic distributions along the gradient of focal class area. Shading indicates the portion of the neutral gradient represented by values in real landscapes. Surface height indicates the mean metric value for 100 replicate runs for each $\mathrm{H} \times \mathrm{P}$ combination. The metric surface grids represent each step in the area and aggregation gradients. Full metric names are provided in Table 1. 

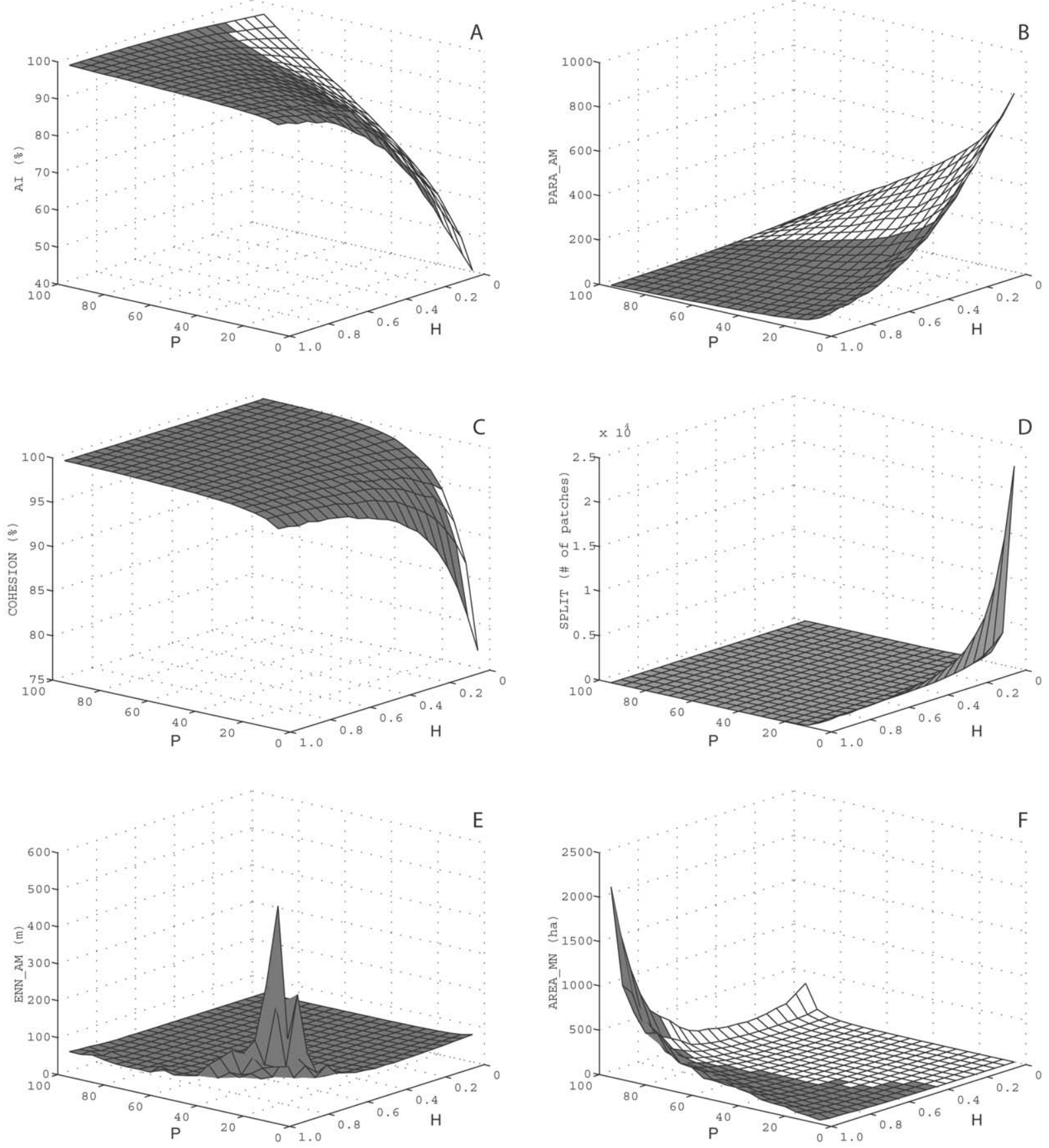

High Density of Real Landscapes

Low Density of Real Landscapes

No Real Landscapes

Figure 6. Six representative metrics from the three strongly non-linear distribution categories. Surface height indicates the mean metric value for 100 replicate runs for each $\mathrm{H} \times \mathrm{P}$ combination. The grid on the metric surface represents each step in the area and aggregation gradients. Shading indicates the portion of the neutral gradient represented by values in real landscapes. Full metric names are provided in Table 1. 
matically nonlinear behavior at extreme values of $\mathrm{H}$ and $\mathrm{P}$ (Figure 6C-F).

\section{Parabolic distributions along the $P$ gradient}

This subgroup included 13 metrics that share a common distributional pattern of a non-linear increase with increasing values of $\mathrm{P}$, typically with a maximum at some intermediate value, and then a subsequent decline (Figure 5). The magnitude of the maximum value of most of these metrics declined with increasing values of $\mathrm{H}$. This decreasing magnitude along the $\mathrm{H}$ gradient also provides at least some measure of class aggregation that is independent of class area. This subgroup of metrics is closely allied with the subgroup of metrics which are strongly related to class aggregation (Figure 4). While the ends of the continuum are distinct, it could be argued that the metrics in these two groups represent a single group of metrics primarily affected by the $\mathrm{H}$ gradient but exhibiting a gradient of responses with respect to their non linearity along the $\mathrm{P}$ gradient, ranging from nearly linear (e.g., PAFRAC) to markedly parabolic (e.g., ED and PD).

While the 13 metrics in this subgroup share the parabolic shape, the point along the area gradient at which maximum values were realized differed (Figure 4). For example, LSI was maximum at $\mathrm{P} \approx 30 \%$; $\mathrm{PD}$ was maximum at $\mathrm{P} \approx 20 \%$ at low $\mathrm{H}$ and at $\mathrm{P}=$ $30 \%-40 \%$ at higher $\mathrm{H}$; ED was maximum and nLSI was minimum at $\mathrm{P}=50 \%$; SHAPE_AM was maximum at approximately $\mathrm{P}=65 \%$; and DCORE_CV peaks at $\mathrm{P}=95 \%$. The value of $\mathrm{P}$ at which DCAD reaches its maximum will vary depending on the edge depth specified, with maximums at higher values of $\mathrm{P}$ as edge depth increases. As edge depth approaches 0, the distribution of DCAD approaches the distribution of PD.

Non-linear behavior across gradients in area makes interpretation of these metrics problematic. In general, they cannot be interpreted alone and require concurrent examination of at least one other metric. For example, ED is typically interpreted in conjunction with number of patches or class area. Also, if the metrics are to be used in further analyses that assume linear relationships (e.g., Pearson product moment correlation or principal components analysis), inclusion of these metrics may be inappropriate. However, if the landscapes being analyzed represent a relatively small proportion of the total $\mathrm{H} \times \mathrm{P}$ gradient, the surface may be approximately planar and thus not violate assumptions of linear relationships. Additionally, parabolic behavior was most pronounced at low $\mathrm{H}$ while values in most real landscapes were comparable to those seen for higher values of $\mathrm{H}$ (Figure 5B).

$\mathrm{ED}$ (or the equivalent total length of edge) is the most commonly used metric in this group in studies quantifying effects of fragmentation. Clearly, however, using ED to quantify the amount of edge per unit area in a landscape could be problematic due to the markedly nonlinear response of the metric. Thus, while edge effects have been demonstrated to be one of the most important consequences of fragmentation (e.g., Demaynadier and Hunter 1998; Gibbs 1998; Bergin et al. 2000; Boulet and Darveau 2000; Burke and Nol 2000; Gehlhausen et al. 2000; Mancke and Gavin 2000; Euskirchen et al. 2001), ED may not be the best way to quantify the effects. Further, recent studies have shown that edge effects are dependent on landscape composition and context (e.g., contrast between focal habitat and adjacent patches and amount of like habitat in the vicinity of fragments) (e.g., Heske et al. 2001). Thus, beyond the problems associated with nonlinear behavior, simplistic measures of amount of edge, such as ED, are likely to be insufficient for assessing edge effects. PD has similar behavior to ED and thus has similar problems in quantifying the effects of fragmentation.

\section{Trend from high $H$ and $P$ to low $H$ and $P$} Four metrics (PARA_AM, PLADJ, AI, CAI_AM) exhibited a slightly nonlinear trend from high $\mathrm{H}$ and $\mathrm{P}$ to low $\mathrm{H}$ and $\mathrm{P}$ (e.g., Figure 6A-B). Thus, these metrics have the interesting property of representing strong interactions of both $\mathrm{H}$ and $\mathrm{P}$. On the one hand, this property makes interpretation of these metrics difficult, because it is impossible to distinguish whether a change in the metric is due to a change in $\mathrm{H}$ or $\mathrm{P}$, or both. Given the importance of distinguishing between habitat area and configuration in the study of habitat fragmentation, metrics that confound the two gradients may have limited utility. On the other hand, simultaneous assessment of changes in $\mathrm{H}$ and $\mathrm{P}$ may be desirable when the distinction between area and configuration is not important. In all cases, real landscapes occupied a subset of neutral landscape space, typically where $H \geq 0.15-0.35$. Thus, the observed non-linear behavior is potentially less severe in real landscapes but it is still an issue.

Two closely related metrics in this group, PLADJ and AI (He et al. 2000), measure the degree of aggregation in a focal class based on like cell adjacencies. The difference between the two metrics is that AI 
standardizes the percent like adjacencies based on the maximum number of adjacencies possible given the class area. These metrics are redundant $(r=0.99, \mathrm{p}$ $<0.001)$ and thus would never be used in the same study. Although AI is standardized to the maximum amount of aggregation possible in a landscape, the metric provides misleading results at $\mathrm{P}>50 \%$ because it does not account for the changing minimum amount of aggregation as $\mathrm{P}$ increases. When $\mathrm{P} \leq 50 \%$, a maximally disaggregated landscape will have no cells with like adjacencies. When $\mathrm{P}>50 \%$, minimum aggregation increases linearly with $\mathrm{P}$ and thus the expected aggregation for any $\mathrm{P}$ increases. Because AI does not account for the adjacencies that necessarily occur when $\mathrm{P}>50 \%$, it overestimates the relative level of aggregation in these landscapes. This problem can be avoided by using a normalized version of AI that accounts for both the minimum and maximum like adjacencies possible for any given P (McGarigal et al. 2002).

$\mathrm{AI}$ is purported to be an effective measure of class aggregation (He et al. 2000). The authors assert that AI is superior to several related metrics (e.g., LSI) because it has linear behavior over gradients of aggregation. Our results, however, demonstrate that AI is not linearly related to the aggregation gradient. The metric is linear along the $\mathrm{P}$ gradient under complete spatial randomness (results not shown) but is strongly nonlinear across all levels of aggregation, particularly at low levels of P. Bogaert (2002) has recently criticized AI as not providing any information that is not already provided by LSI or nLSI (the normalized form of LSI). Our results show that the $\mathrm{He}$ et al. (2000) formulation of AI is not redundant with LSI and is redundant with nLSI only when $\mathrm{P} \leq 50 \%$ (Figure 5, Figure 6). If AI is normalized to account for adjacencies that necessarily occur when $\mathrm{P}>$ $50 \%$, it becomes completely redundant with nLSI.

Strongly nonlinear at extreme values of $H$ and $P$ Eleven metrics were strongly nonlinear at extreme values of $\mathrm{H}$ and $\mathrm{P}$; three subgroups were distinguished from one another based on the portion of the $\mathrm{H}$ by $\mathrm{P}$ gradient in which nonlinear behavior was exhibited (Table 1, Figure 6). Despite obvious differences among subgroups there are several noteworthy observations common to all groups. First, although distributions of these metrics tend to be relatively flat throughout much of the $\mathrm{H}$ by $\mathrm{P}$ space, they exhibit threshold-like behavior in a small portion of the space at extreme values of $\mathrm{H}$ or $\mathrm{P}$ (Figure 6). This behavior can make these metrics difficult to interpret because a unit change in $\mathrm{H}$ or $\mathrm{P}$ does not yield that same unit change in the metric value throughout the $\mathrm{H}$ by $\mathrm{P}$ gradient space. More importantly, the low variability across much of the $\mathrm{H}$ by $\mathrm{P}$ gradient space suggests these metrics will often be of little use in distinguishing among landscapes that actually are quite different in composition and aggregation. The proportion of $\mathrm{H} \times \mathrm{P}$ space represented in real landscapes varied among metrics (Table 1, Figure 6). All or nearly all of the $\mathrm{H} \times \mathrm{P}$ space was represented for six of the metrics (Table 1). For the other metrics the degree of overlap varied along the $\mathrm{P}$ gradient. For example, real landscapes existed throughout the $\mathrm{H}$ gradient for CAI_MN and CORE_MN at low P, but at high P real landscapes were restricted to $\mathrm{H}>0.5$. Areas of problematic metric behavior were represented in real landscapes for all metrics in this category; however, in some cases the most extreme values were not represented (Figure 6). It is interesting that five of the metrics in these three subgroups are mean values of patch-based metrics: AREA, GYRATE, CORE, DCORE, and ENN that are without doubt the most commonly used class-level metrics. This poor performance has been noted by others. For example, Schumaker (1996) found that AREA_MN, CORE_MN, and ENN_MN were poor predictors of modeled dispersal success for Northern Spotted Owls in landscapes with different levels of connectivity among old growth forest patches. This poor performance was attributed to sensitivity to small patches, the effects of which are clearly demonstrated in the neutral landscapes examined here. The other formulations of most of these metrics (e.g., area-weighted means and measures of dispersion) typically behave in manners that will be much more informative. Similarly, in a review of 74 published papers assessing connectivity based on both landscape structure and population level processes, Moilanen and Nieminen (2002) found that Euclidean nearest neighbor distances were rarely correlated with individual abundance, colonization events, or patch occupancy. Given the relative lack of sensitivity of different formulations of the Euclidean nearest neighbor metrics throughout most of the $\mathrm{H}$ by $\mathrm{P}$ gradient, this lack of relationship is not surprising.

COHESION (a measure of the cohesiveness of patches in a landscape based on the ratio between the area-weighted mean patch perimeter-area ratio and the area-weighted mean shape index) is another member of this group that appears to be relatively in- 
variant throughout most of the $\mathrm{H} \times \mathrm{P}$ space. At low $\mathrm{H}$, COHESION is relatively invariant at $\mathrm{P}>0.6$; at higher $\mathrm{H}$, COHESION is mostly invariant throughout the $\mathrm{P}$ gradient. As such, our results suggest that this metric is not likely to be useful for detecting landscape changes when $\mathrm{P}>0.40$. This value coincides with the point at which additional habitat loss results in remaining habitat being broken up into small pieces. Interestingly, Schumaker (1996) found that COHESION did predict modeled spotted owl dispersal and thus was useful in distinguishing among different levels of landscape connectivity. At first this result would seem contrary to the behavior of this metric; however, the percent of the landscapes that were occupied by the focal class in his landscapes ranged from $5-33.7 \%$ (Schumaker 1996) which coincides with the portion of the $\mathrm{H} \times \mathrm{P}$ gradient in which COHESION is sensitive (Figure 6).

The metrics in this group also illustrate that many class-level landscape metrics are not stable, and are therefore difficult to interpret, at extreme values of $\mathrm{H}$ and especially $\mathrm{P}$. This instability is not necessarily a problem with the metrics per se, but rather accentuates the need to understand what the metrics are describing and to apply them intelligently. For example, when the focal class dominates the landscape and forms a matrix, it is not meaningful to measure landscape structure with patch-based metrics. Similarly, when the focal class is extremely rare, patch-based metrics do a poor job of distinguishing among levels of configuration. In addition, extremely low values of $\mathrm{H}$ are not typical in landscapes, thus erratic behavior of metrics under those conditions will most often not be manifested, but our results comparing real and neutral landscape indicate that problematic conditions are possible in real landscapes for a number of metrics (Figure 6).

\section{Conclusions and limitations}

The patterns of behavioral similarities among metrics across the $\mathrm{H}$ by $\mathrm{P}$ space supports several of our expectations. First, the similarities among metrics within each behavioral group suggests that many metrics are closely related, if not perfectly redundant, and describe similar aspects of landscape structure. This point has been shown previously by others ( $\mathrm{Ri}$ itters et al. 1995; Cain et al. 1997) and thus was not surprising. Interestingly though, the cluster analysis and three-dimensional surface plots revealed several close behavioral similarities among metrics that were not intuitively obvious prior to the analysis, despite our intimate familiarity with these metrics. Second, the number of groups of metrics exhibiting markedly different behavior suggests that there are many important dimensions to landscape structure that cannot be succinctly described with only a few metrics. The high dimensionality of landscape structure we observed was especially intriguing because we explicitly varied only two aspects of structure: class area and aggregation. It is likely that additional landscape structure dimensions exist and are more prominent in real landscapes. While there were a number of dimensions of landscape structure, the $\mathrm{H}$ and $\mathrm{P}$ gradients we imposed in our study design were dominant. While this limits the scope of our results, these two gradients are considered to be fundamentally important in real landscapes (Turner 1989; Gustafson 1998).

Our study also provided several new insights into the behavior and utility of landscape metrics. First, landscape structure metrics have traditionally been organized conceptually according to the aspect of landscape composition or configuration they supposedly measure (Table 1). It is common for practitioners to choose metrics from each of the conceptual classes in order to describe different aspects of a landscape (e.g., Ripple et al. 1991). Our results show that it is also important to consider the behavioral groupings because a number of conceptually different metrics have similar behavior and thus are redundant. Similarly, metrics from the same conceptual group often exhibit widely varying behaviors indicating differences in how they respond to attributes of landscape pattern.

Second, our results provide insight into why it has been difficult to find consistent relationships between landscape structure and ecological processes. For example, Schumaker (1996) found that fractal dimension was a poor predictor of modeled dispersal success for Northern Spotted Owls. He indicated that fractal dimension was well behaved in neutral landscapes but behaved unpredictably and non-intuitively in real landscapes. Our results indicate that the lack of relationship between any measure of fractal dimension (PAFRAC, FRAC_AM, FRAC_SD, or FRAC_CV) and modeled spotted owl dispersal should be expected based on the behavior of these metrics in neutral landscapes. Dispersal success was best predicted by metrics that exhibit a strong relationship to class to area (e.g., TCA, CORE_AM, 
SHAPE_AM, PARA_AM, and COHESION) over the range of $\mathrm{P}$ considered in his study (Schumaker 1996). None of the fractal dimension metrics show such relationships. For example, FRAC_AM is parabolic along P (Figure 5), while FRAC_SD (Figure 4),FRAC_CV and PAFRAC are most closely related to the aggregation gradient. Thus, results from neutral landscapes can help in interpreting patterns found in real landscapes.

Third, our results suggest that many metrics purported to have a certain behavior may not behave in the intended manner over the entire $\mathrm{H}$ by $\mathrm{P}$ space. In particular, we found that some metrics purported to be measures of class aggregation (e.g., AI) confounded class area and aggregation (Figure 6). Similarly, most metrics exhibited nonlinear behavior, although the magnitude and pattern of non linearity varied markedly among metrics. Nonlinear behavior renders the interpretation of metric values problematic because a unit change in the metric does not reflect that same degree of change in landscape structure over the range of the metric. Finally, a number metrics exhibit erratic and/or unstable behavior at very high or very low $\mathrm{P}$ and demonstrate that landscape structure is difficult to characterize at the class level when the focal class is either dominant or extremely rare. Fortunately, quantifying configuration may not be that relevant or interesting in such landscapes anyway.

Our findings must be interpreted within the context of several limitations imposed by the use of binary neutral landscapes in our study design. Most importantly, neutral landscapes differ from real landscapes in many important ways. Thus, the beneficial features of neutral landscapes in allowing strict and independent control of certain aspects of landscape structure also mean that many aspects of structure are not reflected. In particular, we evaluated a two-dimensional landscape structure gradient space where configuration was represented solely by class aggregation. Thus, metrics were grouped based on how they behaved in response to changing class area and aggregation, yet many metrics are purported to measure other aspects of landscape configuration. There is no doubt that many of the metrics reported to be partly or wholly redundant in this study would reveal unique behavior in relationship to other gradients of landscape configuration. Unfortunately, controlling class configuration in neutral landscapes is extremely limited with available software; generating a broader array of configurations remains an important focus for future software development. In addition, because we used binary landscapes, many metrics that characterize important aspects of landscape structure in real landscape (e.g., edge contrast, similarity, interspersion, and diversity) were not relevant and we could not adequately evaluate their behavior. Similarly, landscape-level metrics were not meaningful to examine so our results pertain only to class-level metrics. While the binary nature of the landscapes limited the range of metrics we could evaluate, it does not limit the applicability of these results in landscapes with more than two classes. The classlevel metrics we present here pertain to the focal class and ignore the class identities and patterns of the surrounding mosaic (i.e., the metrics take an island biogeographic perspective). This island biogeographic perspective is commonly used in fragmentation studies because fragmentation is inherently a phenomenon that is relevant in context of a focal class. Further differences between real and neutral landscapes that limit the application of these results that neutral landscapes tend to have larger numbers patches, particularly of extremely small patches, than real landscapes while larger patches (especially at higher P values) tend to be more extensive and convoluted than larger patches in real landscapes. These differences result in values of many metrics from real landscapes falling outside the space occupied by neutral landscapes. Obviously, our results can most reliably be applied to real landscapes when metric values either mostly overlap with values from neutral landscapes or occupy a subset of values occupied by neutral landscapes (groups 1 and 4, Table 1).

Our results are also limited because we examined only one scale of observation (i.e., grain and extent were the same for all neutral landscapes). Relationships of metric responses to varying grain and extent are known to be complex, with both the metric values and distributional shape being affected (e.g., O'Neill et al. 1996; Saura 2002; Wu et al. 2002). For example, in an investigation involving real landscapes, Wu et al. (2002) found that metrics fell into three categories of response to changing scale; responses were predictable in only one of these categories. Thus, while we are aware of the importance of scale, conducting a multi-scale investigation was beyond the scope of the present investigation and thus our results and conclusions are limited to the scale of our examination.

In conclusion, while landscape metrics have proven useful for describing landscape structure and hold 
promise for broader application, they are often difficult to interpret. More importantly, links between ecological processes and landscape pattern as measured by these metrics have sometimes been difficult to demonstrate (Schumaker 1996; Moilanen and Nieminen 2002). We argue that understanding expected metric behavior can aid in selecting and interpreting metrics that are sensitive to changes resulting from a phenomenon of interest.

\section{Acknowledgments}

We thank M. Cummings and D. Myers of the University of Maryland for assistance with Perl programming. This work was supported in part by a David H. Smith Conservation Research Postdoctoral fellowship to MCN and a GAAN graduate fellowship through United States Department of Education to SAC. This material is based on work partially supported by the Cooperative State Research, Extension, Education Service, U.S. Department of Agriculture, Massachusetts Agricultural Experiment Station and the Department of Natural Resources Conservation, under Project No. 3322. This paper is Publication no. DHS2003-05 of the Nature Conservancy's David H. Smith Conservation Research Fellowship Program.

\section{References}

Belisle M. and Clair C.C.S. 2002. Cumulative effects of barriers on the movements of forest birds. Conservation Ecology 5: 480-495.

Bender D.J., Contreras T.A. and Fahrig L. 1998. Habitat loss and population decline: A meta-analysis of the patch size effect. Ecology 79: 517-533.

Bergin T.M., Best L.B., Freemark K.E. and Koehler K.J. 2000. Effects of landscape structure on nest predation in roadsides of a midwestern agroecosystem: a multiscale analysis. Landscape Ecology 15: 131-143.

Bogaert J. 2002. A mathematical comment on the formulae for the aggregation index and the shape index. Landscape Ecology 17: 87-90.

Boulet M. and Darveau M. 2000. Depredation of artificial bird nests along roads, rivers and lakes in a boreal balsam fir, Abies balsamea, forest. Canadian Field-Naturalist 114: 83-88.

Burke D.M. and Nol E. 2000. Landscape and fragment size effects on reproductive success of forest-breeding birds in Ontario. Ecological Applications 10: 1749-1761.

Cain D.H., Riitters K. and Orvis K. 1997. A multi-scale analysis of landscape statistics. Landscape Ecology 12: 199-212.

Chen J., Franklin J.F. and Spies T.A. 1995. Growing season microclimatic gradients from clear cut edges in old-growth Douglasfir forests. Ecological Applications 5: 74-86.
Demaynadier P.G. and Hunter J.L.J. 1998. Effects of silvicultural edges on the distribution and abundance of amphibians in Maine. Conservation Biology 12: 340-352.

Euskirchen E.S., Chen J.Q. and Bi R.C. 2001. Effects of edges on plant communities in a managed landscape in northern Wisconsin. Forest Ecology and Management 148: 93-108.

Fahrig L. 1998. When does fragmentation of breeding habitat affect population survival? Ecological Modelling 105: 273-292.

Fahrig L. 2002. Effect of habitat fragmentation on the extinction threshold: A synthesis. Ecological Applications 12: 346-353.

Fahrig L. and Jonsen I. 1998. Effect of habitat patch characteristics on abundance and diversity of insects in an agricultural landscape. Ecosystems 1: 197-205.

Fahrig L. and Merriam G. 1985. Habitat patch connectivity and population survival. Ecology 66: 1762-1768.

Gardner R.H. 1999. RULE: Map generation and a spatial analysis program.. In: Klopatek I. and Gardner R.H. (eds), Landscape Ecological Analysis: Issues and Applications, pp. 280-303. Springer-Verlag, New York, New York, USA.

Gardner R.H., Milne B.T., Turner M.G. and O’Neill R.V. 1987. Neutral models for the analysis of broad-scale landscape patterns. Landscape Ecology 1: 19-28.

Gehlhausen S.M., Schwartz M.W. and Augspurger C.K. 2000. Vegetation and microclimate edge effects in two mixedmesophytic forest fragments. Plant Ecology 147: 21-35.

Gibbs J.P. 1998. Amphibian movements in response to forest edges, roads, and streambeds in southern New England. Journal of Wildlife Management 62: 584-589.

Gustafson E.J. 1998. Quantifying landscape spatial pattern: What is the state of the art? Ecosystems 1: 143-156.

Gustafson E.J. and Parker G.M. 1992. Relationships between land cover proportion and indices of landscape spatial pattern. Landscape Ecology 7: 101-110.

Hargis C.D., Bissonette J.A. and David J.L. 1997. Understanding measures of landscape pattern. In: Bissonette J.A. (ed.), Wildlife and Landscape Ecology, pp. 231-261. Springer-Verlag, New York, New York, USA.

Hargis C.D., Bissonette J.A. and David J.L. 1998. The behavior of landscape metrics commonly used in the study of habitat fragmentation. Landscape Ecology 13: 167-186.

Haydon D.T. and Pianka E.R. 1999. Metapopulation theory, landscape models, and species diversity. Ecoscience 6: 316-328.

He H.S., DeZonia B.E. and Mladenoff D.J. 2000. An aggregation index (AI) to quantify spatial patterns of landscapes. Landscape Ecology 15: 591-601.

Heske E.J., Robinson S.K. and Brawn J.D. 2001. Nest predation and neotropical migrant songbirds: piecing together the fragments. Wildlife Society Bulletin 29: 52-61.

Iverson L.R. 1989. Land use changes in Illinois, USA: the influence of landscape attributes on current and historic land use. Landscape Ecology 2: 45-61.

Jaeger J.A.G. 2000. Landscape division, splitting index, and effective mesh size: new measures of landscape fragmentation. Landscape Ecology 15: 115-130.

Kareiva P. and Wennergren U. 1995. Connecting Landscape Patterns to Ecosystem and Population Processes. Nature 373: 299302.

Keitt T.H., Urban D. and Milne B.T. 1997. Detecting critical scales in fragmented landscapes. Conservation Ecology 1: 4. 
Krummel J.R., Gardner R.H., Sugihara G., O’Neill R.V. and Coleman P.R. 1987. Landscape patterns in a disturbed environment. Oikos 48: 321-324.

Li H. and Reynolds J.F. 1993. A new contagion index to quantify spatial patterns of landscapes.Landscape Ecology 8: 155-162.

Mancke R.G. and Gavin T.H.. 2000. Breeding bird density in woodlots: effects of depth and buildings at the edges. Ecological Applications 10: 598-611.

McGarigal K. 2002. Landscape pattern metrics. In: El-Shaarawi A.H. and Piegorsch W.W. (eds), Encyclopedia of Environmentrics, Volume 2, pp. 1135-1142. John Wiley \& Sons, Sussex, England.

McGarigal K., Cushman S.A., Neel M.C. and Ene E. 2002. FRAGSTATS: Spatial Pattern Analysis Program for Categorical Maps. Computer software program produced by the authors at the University of Massachusetts, Amherst, Massachusetts, USA. Available at the following web site:www.umass.edu/landeco/ research/fragstats/fragstats.html.

McGarigal K. and Marks B.J. 1995. FRAGSTATS: a spatial pattern analysis program for quantifying landscape structure. USDA Forest Service. GTR PNW-351.

McGarigal K. and McComb W.C. 1995. Relationships between landscape structure and breeding birds in the Oregon Coast Range. Ecological Monographs 65: 235-260.

Merriam D.F. and Sneath P.H.A. 1966. Quantitative comparison of contour maps. Journal of Geophysical Research 71: 1105-1115.

Milne B.T. 1988. Measuring the fractal geometry of landscapes. Applied Mathematics and Computation 27: 67-79.

Moilanen A. and Nieminen M. 2002. Simple connectivity measures in spatial ecology. Ecology 83: 1131-1145.

O’Neill R.V., Hunsaker C.T., Jones K.B., Riitters K.H., Wickham J.D., Schwartz P.M., Goodman I.A., Jackson B.L. and Baillargeon W.S. 1997. Monitoring environmental quality at the landscape scale. BioScience 47: 513-519.

O’Neill R.V., Hunsaker C.T., Timmins S.P., Timmins B.L., Jackson K.B., Jones K.B., Riitters K.H. and Wickham J.D. 1996. Scale problems in reporting landscape pattern at the regional scale. Landscape Ecology 1: 169-180.

Riitters K., Wickham J., O'Neill R., Jones B. and Smith E. 2000. Global-scale patterns of forest fragmentation. Conservation Ecology 4: 27-56.

Riitters K.H., O’Neill R.V., Hunsaker C.T., Wickham J.D., Yankee D.H., Timmins S.P., Jones K.B. and Jackson B.L. 1995. A factor analysis of landscape pattern and structure metrics. Landscape Ecology 10: 23-39.

Ripple W.J., Bradshaw G.A. and Spies T.A. 1991. Measuring forest landscape patterns in the Cascade Range of Oregon, USA. Biological Conservation 57: 00-00.

Robinson A.H. 1962. Mapping the correspondence of isarithmic maps. Annals of the Association of American Geographers 52: $414-425$.
Robinson A.H. and Bryson R.A. 1957. A method for describing quantitatively the correspondence of geographical distributions. Annals of the Association of American Geographers 47: 379-391.

SAS Institute 1999. SAS, Version 8. 01. SAS Institute Incorporated, Cary, North Carolina, USA.

Saupé D. 1988. Algorithms for random fractals.. In: Peitgen H.- O. and Saupé D. (eds), The Science of Fractal images, pp. 71-113. Springer-Verlag, New York, New York, USA.

Saura S. 2002. Effects of minimum mapping unit on land cover data spatial configuration and composition. International Journal of Remote Sensing 23: 4853-4880.

Saura S. and Martínez-Millán J. 2000. Landscape patterns simulation with a modified random clusters method. Landscape Ecology 15: 661-678.

Schumaker N.H. 1996. Using landscape indices to predict habitat connectivity. Ecology 77: 1210-1225.

Stauffer D.F. 1985. Introduction to Percolation Theory. Taylor and Francis, London, UK.

The MathWorks Inc. 2001. MATLAB, Natick, Massachusetts, USA.

Trzcinski M.K., Fahrig L. and Merriam G. 1999. Independent effects of forest cover and fragmentation on the distribution of forest breeding birds. Ecological Applications 9: 586-593.

Turner M.G. 1989. Landscape ecology: the effect of pattern on process. Annual Review of Ecology and Systematics 20: 171197.

Turner M.G. and Ruscher C.L. 1988. Changes in the spatial patterns of lands use in Georgia. Landscape Ecology 1: 241-251.

Wickham J.D., Jones K.B., Riitters KH., Wade T.G. and O'Neill R.V. 1999. Transitions in forest fragmentation: implications for restoration opportunities at regional scales. Landscape Ecology 14: 137-145.

Wickham J.D., Riitters K.H., O’Neill R.V., Reckhow K.H., Wade T.G. and Jones K.B. 2000. Land cover as a framework for assessing risk of water pollution. Journal of the American Water Resources Association 36: 1417-1422.

With K.A., Gardner R.H. and Turner M.G. 1997. Landscape connectivity and population distributions in heterogeneous environments. Oikos 78: 151-169.

With K.A. and King A.W. 2001. Analysis of landscape sources and sinks: the effect of spatial pattern on avian demography. Biological Conservation 100: 75-88.

Wu J.G., Shen W.J., Sun W.Z. and Tueller P.T. 2002. Empirical patterns of the effects of changing scale on landscape metrics. Landscape Ecology 17: 761-782. 